\title{
Percutaneous aortic valve replacement: Which patients are suitable for it? A quest for a controlled use
}

Francesca di Marco, MD, and Gino Gerosa, MD

See related editorial on page 299.

From the Department of Cardiac, Thoracic, and Vascular Sciences, Padua University Medical School, Division of Cardiac Surgery, Padova, Italy.

Received for publication July 25, 2006; accepted for publication Aug 8, 2006.

Address for reprints: Gino Gerosa, MD, Division of Cardiac Surgery, Department of Cardiac, Thoracic, and Vascular Sciences, Padua University Medical School, Via Giustiniani 2, 35100 Padova, Italy (E-mail: gino.gerosa@unipd.it)

J Thorac Cardiovasc Surg 2007;133:294-8

$0022-5223 / \$ 32.00$

Copyright (C) 2007 by The American Association for Thoracic Surgery

doi:10.1016/j.jtcvs.2006.08.074
7 he internal thoracic artery-to-left descending coronary artery bypass graft $(\mathrm{CABG})$ is recognized as the gold standard therapy for coronary artery disease. Nevertheless, percutaneous revascularization is currently adopted as the first-line approach, not only in the treatment of multivessel disease but even in the presence of left main stenosis and in patients with diabetes. Although no major randomized trials justify this policy, still it has been currently adopted. ${ }^{1}$ Many reasons might explain the widespread embracement of endovascular techniques over surgery. In one word: simplification. The patient does not need to be sedated or intubated. The duration of the procedure is relatively short, and the results can be ex tempore visualized, allowing a possible adjustment. A very short hospitalization is required for recovery, and the patient's stress is minimized. Finally, the procedures are easily repeatable, in contrast to the widely known risks of a resternotomy.

Just because of their simplified character, a wide number of interventional procedures are performed every day compared with cardiac operations, allowing the catheter technology to evolve rapidly: new techniques are launched on the market with an impressive speed and are applied in the catheterization laboratories with a quick turnover. By contrast, apart from spare attempts (ministernotomy, port-access technique, robotics), cardiac surgery techniques remain substantially unchanged since their introduction.

Valve surgery has long represented a stronghold for the cardiac surgeon, but things might change. In fact, although the research on the ideal valve substitute is still ongoing, endovascular techniques have recently been proposed as alternative therapies for both mitral and aortic disease. The attempt to renew valve surgery with the introduction of robotics for mitral valve surgery has actually complicated it. In fact, even if robotic surgery can minimize patient trauma and promote a faster recovery, it is associated with higher use of resources, in both economic and human terms. Even if surgery can be realized without opening the chest, robotics is not synonymous with simplification, whereas a percutaneous technique remains a simplified technique when compared with surgery.

The opportunity to replace an aortic valve in the awake patient by a vascular access is undoubtedly appealing both for the patient and the physician, but before an uncontrolled diffusion of endovascular aortic valve replacement (AVR) occurs, mimicking the widespread preference for percutaneous revascularization over $\mathrm{CABG}$, we should ask ourselves these questions:

1. What are the current results of surgical AVR in terms of in-hospital morbidity and mortality?

2. Is percutaneous AVR effective?

3. Are we sure that the risks related to valve surgery are greater than the risks of an endovascular procedure? In fact, simple does not necessarily mean safe and durable; on the other hand, surgery does not necessarily imply a significant risk.

4. The clinical application of percutaneous AVR has started on patients excluded from cardiac surgery; currently, which patients would be excluded from surgery after due consideration and why?

The analysis of The Society of Thoracic Surgeons National Cardiac Database evidenced that, among 46,397 patients, mortality for AVR ranges from $4.3 \%$ for first isolated AVR to $25 \%$ for redo surgery or multiple valve replacement plus CABG, 
with an overall rate of $6.4 \% .^{2}$ In a very impartial editorial, Fish $^{3}$ outlines that the surgical results are excellent even in high-risk patients and, for this reason, percutaneous approaches must (1) justify their adoption and (2) guarantee high standards for performance.

Nevertheless, in minor and tendentious reports, ${ }^{4}$ surgical mortality is considered prohibitive in certain categories of patients, which are vaguely described as " at high risk," and alternative solutions (interventional, of course) are "needed." On the contrary, we believe that the adoption of percutaneous approaches, even in selected cases, needs to be justified.

According to the current results of surgical AVR at our institution between December 2002 and June 2006, 341 consecutive patients (mean age $70 \pm 11$ years, median age 73 years, range 23-89 years) underwent isolated AVR. Fifty-three (15\%) patients were 80 years old or older, and $9.6 \%$ of those patients had previous cardiac surgery; in particular, 3.2\% had previous CABG surgery, with a patent left internal thoracic artery-to-left descending coronary artery graft. The patients had multiple comorbidities (in $80 \%$ of patients, 3 risk factors coexisted; in 55\% of patients, 5 coexisted). Seven percent of patients were affected by chronic renal failure (serum creatinine $>150 \mathrm{mg} / \mathrm{dL}$ ), $10 \%$ had previous stroke, $17 \%$ of patients had diabetes, and $25 \%$ were affected by chronic lung disease. In $8 \%$ the left ventricular function was severely impaired, with an ejection fraction less than $40 \%$. Finally, $2 \%$ of patients required an urgent operation. The operative risk, which was calculated by EuroSCORE grading, ${ }^{5}$ was low (EuroSCORE $0-2$ ) in $24 \%$ of patients and medium (EuroSCORE 3-5) in $46 \%$. In $30 \%$ of patients the operative risk was high (EuroSCORE $\geq 6$ ). According to in-hospital morbidity, major neurologic complications (either stroke or transient ischemic attack) occurred in $0.9 \%$ of patients, and acute renal failure and respiratory failure occurred in $0.3 \%$ of patients. In $4 \%$ of patients, postoperative bleeding required a surgical revision, and perioperative acute myocardial infarction was observed in $0.6 \%$. Overall in-hospital mortality was $0.3 \%$, whereas the expected mortality (according to a mean EuroSCORE of 4.3) was 3\%. Among this population, some challenging situations were encountered: severe calcifications of the ascending aorta were observed in 7 patients and an intraoperative conversion of the surgical strategy to a Bentall operation was necessary in 3 of them. Three patients with a porcelain aorta were scheduled to undergo apicoaortic bypass surgery instead of traditional AVR.

Sixty-nine percent of patients received a biologic prosthesis, whereas in $31 \%$ a mechanical valve was implanted. Mean follow-up time was $16 \pm 11$ months. Kaplan-Meier survival was $92 \% \pm 0.03 \%$ at 40 months.

In view of the results of percutaneous AVR, the optimism expressed is premature. In fact, the only published series (6 patients affected by end-stage aortic stenosis), presented by Cribier and associates, ${ }^{6}$ evidenced some major drawbacks, such as perivalvular leakage, which is caused by the persistence of empty space between the percutaneous and native valves owing to calcifications and which was observed in the majority of patients. Moreover, coronary flow obstruction provoked by the valved stent and atheroembolism of calcific debris during the positioning of the device are possible. Grube and colleagues ${ }^{4}$ have recently described 1 single case of implantation of a self-expandable valve prosthesis by the retrograde approach, which was deemed to facilitate coaxial positioning and to reduce the risk of perivalvular leakage, but required extracorporeal circulatory support (ECC) as a "safety measure." In fact, some intraprocedural complications, such as ejection of the valve into the ascending aorta at the time of balloon inflation or hemodynamic collapse after balloon predilation, have been reported. ${ }^{6}$ Finally, several technical difficulties have been described for both the antegrade and retrograde approach, ${ }^{4}$ so that a transapical access through a median sternotomy has been hypothesized. However, we are concerned that a percutaneous AVR that necessitates either a median sternotomy or ECC no longer sounds like percutaneous AVR.

The technique has been defined by several authors as "attractive" and "promising," being able in the near future to oust aortic valve surgery. However, we believe that the evidenced limits make it not so attractive and that this approach is not so promising, especially for insufficient aortic valves, which might not be suitable at all for percutaneous replacement. In fact, in the vast majority of cases, the insufficiency is due to a degenerative disease that usually involves the entire aortic root, with subsequent dilation and an increased risk for potential perivalvular leakage. Besides, an unacceptable risk of coronary flow obstruction might be the consequence of the pathophysiologic expansion of the left ventricular outflow tract characterizing aortic insufficiency. ${ }^{3}$

Before being accepted, transcatheter valve replacement needs to prove its effectiveness when compared with surgical valve replacement, which is and remains the gold standard.

The first human report of percutaneous AVR by Cribier and colleagues ${ }^{7}$ dates back to 2002 and described a single patient who died 17 weeks after the implant of complications related to peripheral artery disease. More recently, the same group ${ }^{6}$ reported their overall clinical experience: among 11 patients with end-stage aortic stenosis, they encountered intraprocedural technical failures in 2 (18\%) patients and a high short-term mortality resulting from comorbidities (4 patients). At a very short follow-up time (18 days3.5 months), there were no signs of heart failure in the remaining 6 patients. Grube and coworkers ${ }^{4}$ have recently proposed an antegrade transseptal implantation, supported by ECC, of a 
self-expandable CoreValve aortic valve (CoreValve SA, Paris, France). They reported a 2-week follow-up with no complications, specifying that the only patient treated (who was presumably too impaired to withstand a surgical AVR) will be followed up during the next 5 years. Their intentions are good, but probably not realistic. Furthermore, we believe that any surgical report with a 2 -week follow-up can reasonably be judged inadequate.

What about the guarantees of functioning and durability offered by valved stents? Cribier and associates ${ }^{7}$ developed an original percutaneous valve initially composed of three bovine pericardial leaflets mounted within a balloonexpandable stent; the valve had passed 2 million cycles, corresponding to $2 \frac{1}{2}$ years. In their most recent experience, they ${ }^{6}$ adopted a newly designed valve formed by equine pericardial leaflets mounted within a reinforced stent that had completed 200 million cycles. Before clinical implant, both valves were tested in animal models. However, we share the concerns of Fish $^{3}$ about the possible unsuitability of animal models for preclinical evaluation of percutaneous valves, inasmuch as the results are strictly dependent on the native valve status. We strongly believe that current Food and Drug Administration requirements need an adjustment for percutaneous devices in consideration of the specific features of both the devices and the procedural methods.

Percutaneous treatment currently does not offer adequate guarantees inasmuch as the preclinical evaluation is potentially inadequate and the clinical experience is poorly substantiated by short-term results. By contrast, the limits of interventional approaches appear clear ${ }^{8}$ and mainly represented by the following issues:

- Current approaches and delivery routes are unsatisfactory: currently, both antegrade and retrograde approaches have been proposed, but none appear to be free from disadvantages.

- Optimal positioning of the stent to avoid coronary ostial impairment and paravalvular leakage is still a challenge.

The need for an optimal approach is evidenced also by the recent attempts to perform hybrid procedures, ${ }^{9}$ which require a ministernotomy or epigastric incision to deliver a sutureless valve through the cardiac apex. The technical difficulties in the optimal positioning of the valved stent have been overcome by some authors with the support of ECC, ${ }^{4}$ which partially negates the utility of a nonsurgical strategy. The possible jeopardizing of coronary flow is still a major challenge in insufficient aortic valves owing to the specific pathophysiologic anatomic modifications. ${ }^{3}$ Finally, paravalvular leakage was evident in 5 of 6 patients in the experience reported by Cribier and colleagues ${ }^{6}$ (mild in 3 and severe in 2 patients); the risk of compromising hemodynamics results in severely ill patients.
Finally, we must be aware that cardiac surgeons themselves might contribute to the currently inappropriate spreading of percutaneous techniques. In fact, the analysis of a nationally representative database ${ }^{2}$ has outlined that a significant portion of patients affected by severe aortic stenosis are not even considered for aortic valve surgery. In fact, they are considered to be at prohibitive operative risk because of either significant comorbidities or advanced age. We should consider this information as an alert, because this cohort of patients might be inappropriately labeled as inoperable and arbitrarily referred to interventional cardiologists for a percutaneous approach. In a recent series published by Gammie and collegues, ${ }^{10}$ among 14 patients undergoing aortic valve bypass surgery as alternative surgery for aortic stenosis, $50 \%$ of patients had been initially excluded from surgery in other centers. The question is, 10 years ago these patients would have been treated with the sole medical therapy, but what about now? How many of those might be referred for percutaneous AVR as inappropriate compassionate therapy?

We believe that the factors that in the past were traditionally considered as contraindications for cardiac surgery no longer contraindicate AVR; instead, they must be considered as factors that make the operation more challenging and require tailored surgical strategies. Mainly, four issues need to be deepened:

1. Reduced life expectancy owing to either advanced age or significant commorbidities

2. Presence of heavy calcifications of the ascending aorta, known as porcelain aorta

3. Previous $\mathrm{CABG}$ with patent grafts

4. Pathologic conditions that contraindicate cardiopulmonary bypass

Regarding reduced life expectancy, the old paradigm that considered AVR worthwhile only in those patients whose life expectancy was equal or superior to the expected durability of the prosthesis implanted has been overcome. As a consequence of continuous improvements in both intraoperative and postoperative care, operative mortality rates have been lowered to $0.3 \%$ for isolated AVR, as shown by our experience. The very low perioperative risk along with the widely known unfavorable natural history of severe aortic stenosis contributed to the extension of surgical indications to octogenarian and even nonagenarian patients. Currently, advanced age cannot be considered an exclusion criterion for AVR, and nonagenarian patients have successfully undergone valve surgery. ${ }^{11}$

Porcelain aorta is a rarely encountered condition, being characterized by a severely calcified ascending aorta that makes aortic clamping extremely difficult or impossible and exposes the patient to the risk of neurologic deficits secondary to atheroembolization and postoperative aortic dissection. Deep hypothermic circulatory arrest with replacement of the ascending aorta has been proposed, ${ }^{12}$ but simplified 
methods such as apicoaortic conduit and endovascular clamping appear as more appealing alternatives. An apicoaortic conduit that consists in the interposition of a valved graft between the apex of the left ventricle and the descending aorta was first evaluated clinically about 50 years ago ${ }^{13}$ but soon was abandoned because of high rates of hemolysis and embolization compared with conventional AVR.

The recent introduction of transcatheter approaches, proposed for those patients excluded from surgery because of porcelain aorta, induced cardiac surgeons to reinterpret the apicoaortic conduit as an alternative to conventional surgery able to overcome the limits imposed by a severely calcified aorta. In this view, apicoaortic conduit surgery offers several advantages:

- It does not require sternotomy, but can be realized through a lateral thoracotomy. Minithoracotomy associated with thoracoscopy has been also reported. This is particularly useful in those patients who undergo a resternotomy and especially in patients with patent coronary artery grafts. Besides, it allows faster recovery.

- No damage to native coronary arteries is possible and there is no risk of atrioventricular block.

- There is no risk for cerebral atheroembolization.

- Finally, it can be performed without cardiopulmonary bypass and cardioplegic arrest, with an off-pump technique.

Concomitant carotid artery disease, chronic renal failure, depressed left ventricular systolic function, and other comorbidities that make the candidate unsuitable for conventional on-pump surgery no longer represent an absolute contraindication.

In his series, Gammie and associates ${ }^{10}$ report excellent results of aortic valve bypass surgery in patients otherwise excluded from surgery, with neither postoperative stroke nor permanent renal failure. Those patients, accounting for $5.8 \%$ of all the patients undergoing isolated AVR, were at high operative risk because of prior CABG with patent grafts (12/14 patients), chronic renal failure, severe lung disease, or cerebrovascular disease.

Last, endovascular aortic clamping has been presented as a further no-clamping approach. Ooi and colleagues ${ }^{14}$ developed aortic cannulation by the Seldinger technique plus internal occlusion of the ascending aorta by means of a Foley catheter. The method allows both antegrade administration of cardioplegia and proximal aortic venting.

In conclusion, we believe that currently there are no factors that might preclude surgery for any patient. On the other hand, we are concerned that a percutaneous approach, which has not yet provided enough guarantees compared with surgical therapy, might appear a safer and less demanding alternative both for the patients and for the referring cardiologists.

Instead of finding a way to replace surgical therapy, percutaneous approaches should focus on coming up to the side of surgery, to support it in its current limitations. In fact, even if surgical AVR represents the gold standard therapy for aortic valve disease, the search for the ideal valve substitute is still ongoing: biologic prostheses are associated with unsatisfactory durability in young patients, exposing them to the need for repeated reoperations, and mechanical valves necessitate lifelong anticoagulation therapy. Besides, both of them are inadequate in patients in whom a height-weight growth is expected, and neither homografts nor autografts are satisfying alternatives. We believe that the application of a percutaneous technique, which is easily repeatable, might efficaciously join cardiac surgery in young patients: in these patients the implantation of a bioprosthesis by surgery might be appealing as a first-line approach, since it does not expose the patients to the risks related to anticoagulation. Owing to the limited durability of biologic valves in young patients and the lack of growth potential of artificial valves, further procedures are likely to be necessary. We believe that these procedures might be performed with a percutaneous technique that could be repeated over the years. In fact, the presence of a rigid bioprosthetic stent as well as the absence of calcifications of the degenerated bioprosthesis reduce the risks of periprosthetic leak (which represents a major risk for calcific stenosis).

This hybrid approach would allow young patients to reach the cutoff age for the definitive surgical implantation of a second bioprosthesis without exposing them to the risk of multiple sternotomies.

\section{References}

1. Rankin JS, Harrel FE. Measuring the efficacy of coronary revascularization: implications for future management. J Thorac Cardiovasc Surg. 2006;131:944-8.

2. Astor BC, Kaczmarek RG, Hefflin B, Daley WR. Mortality after aortic valve replacement: results from a nationally representative database. Ann Thorac Surg. 2000;70:1939-45.

3. Fish RD. Percutaneous heart valve replacement. Enthusiasm tempered. Circulation. 2004;110:1876-8.

4. Grube E, Laborde JC, Zickman B, Gerckens U, Federhoff T, Sauren B, et al. First report on a human percutaneous transluminal implantation of a self-expanding valve prosthesis for interventional treatment of aortic valve stenosis. Catheter Cardiovasc Interv. 2005;66:465-9.

5. Kawachi Y, Nakashima A, Toshima Y, Arinaga K, Kawano H. Risk stratification analysis of operative mortality in heart and thoracic aorta surgery: comparison between Parsonnet and EuroSCORE additive model. Eur J Cardiothorac Surg. 2001;20:961-6.

6. Cribier A, Eltchaninoff H, Tron C, Bauer F, Agatiello C, Sebagh L, et al. Early experience with percutaneous transcatheter implantation of heart valve prosthesis for the treatment of end-stage inoperable patients with calcific aortic stenosis. J Am Coll Cardiol. 2004;43:698-703.

7. Cribier A, Eltchaninoff H, Bash A, Borenstein N, Tron C, Bauer F, et al. Percutaneous transcatheter implantation of an aortic valve prosthesis for calcific aortic stenosis. First human case description. Circulation. 2002;106:3006-8.

8. Lutter G, Ardehali R, Cremer J, Bonhoeffer P. Percutaneous valve replacement: current state and future prospects. Ann Thorac Surg. 2004;78:2199-206.

9. Pawelec-Wojtalic M, Antosik P, Wasiatycz G, Wojtalic M. Use of muscular VSD Amplatzer occluder for closing right ventricular free 
wall perforation after hybrid procedure. Eur J Cardiothorac Surg. 2004;26:1044-6.

10. Gammie JS, Brown JW, Brown JM, Poston RS, Pierson RN, Odonkor $\mathrm{PN}$, et al. Aortic valve bypass for the high risk patient with aortic stenosis. Ann Thorac Surg. 2006;81:1605-10.

11. Alexander KP, Anstrom KJ, Muhlbaier LH, Grosswald RD, Smith PK, Jones RH, et al. Outcomes of cardiac surgery in patients aged $\geq 80$ years: results from the National Cardiovascular Network. J Am Coll Cardiol. 2000;35:731-8.
12. Byrne JG, Aranki SF, Cohn LH. Aortic valve operations under deep hypothermic circulatory arrest for the porcelain aorta: "no-touch" technique. Ann Thorac Surg. 1998;65:1313-5.

13. Sarnoff SJ, Donovan TJ, Case RB. The surgical relief of aortic stenosis by means of apical-aortic valvular anastomosis. Circulation. 1955;11: 564-75.

14. Ooi A, Iyenger S, Langley SM, Haw MP. Endovascular clamping of porcelain aorta in aortic valve surgery using Foley catheter. Heart Lung Circ. 2006;15:194-6. 